\title{
THE CALCULUS OF VARIATIONS AND MATERIALS SCIENCE
}

\author{
$\mathrm{BY}$ \\ J. M. BALL \\ Mathematical Institute, University of Oxford, 24-29 St. Giles, Oxford OX1 3LB, U.K.
}

\begin{abstract}
A review is given of the development and present state of the calculus of variations, starting from the problem of the brachistochrone, and emphasizing the current interaction with problems of materials science.
\end{abstract}

1. Introduction. The beginnings of the calculus of variations go back at least as far as the study of isoperimetric problems by the school of Pappus in ancient Greece. However, the start of the modern era of the subject three centuries ago can be marked by the solution by Johann Bernoulli in 1696 of the brachistochrone problem first formulated by Galileo, that of finding the shape of the curve joining two prescribed points in space, down which a particle will slide without friction in the least time. Bernoulli circulated the problem to various mathematicians, among whom Leibniz (in June 1696), Bernoulli's brother Jakob and L'Hospital responded, also correctly identifying the brachistochrone as an arc of a cycloid. Isaac Newton learned of Bernoulli's challenge on the afternoon of 29 January 1697, on returning at 4 p.m. tired after his day's work as Master of the Royal Mint. He had solved the problem, together with another geometrical one posed by Bernoulli, by 4 a.m. the next morning, and the same day communicated his solutions anonymously to the Royal Society. An impressive feat, although Bernoulli later wrote that neither problem should take "a man capable of (solving) it more than half an hour's careful thought" [29] and they took Sir Isaac 12 hours, a warning from the past of how administration dulls the mind! (For an authoritative account of the history see Whiteside [53].)

The subsequent development of the calculus of variations has been an exciting one, and has led in particular to a good, though not exhaustive, understanding of one-dimensional problems. In contrast, for multi-dimensional problems many basic questions remain unresolved. Many of these questions have been illuminated by a fertile interaction in the last 20 years with problems arising from solid mechanics and materials science. I will approach the multi-dimensional issues from a historical perspective, starting from a discussion of the brachistochrone and some highlights of the one-dimensional theory.

Received May 1, 1998.

1991 Mathematics Subject Classification. Primary 49-02, 49K05, 49K20, 49N60, 73-02, 73C50, 73G05, $73 \mathrm{~S} 10$.

E-mail address: ball@maths.ox.ac.uk 
The discussion makes no attempt to be complete, so that many interesting developments are not mentioned, and is inevitably coloured by my own interests.

2. The Brachistochrone. We make the simplifying assumptions that the wire is in the $(x, y)$-plane, with the $x$-axis horizontal and the $y$-axis vertically downwards, and is in the form of a graph $y=y(x)$ joining the origin with the point $x=x_{1}>0, y=y_{1}>0$. The particle starts at the origin with zero velocity. The problem is thus to find the curve $y$ minimizing the time of travel

$$
T(y)=\int_{0}^{x_{1}} \sqrt{\frac{1+y_{x}^{2}}{2 g y}} d x,
$$

where $g$ is the acceleration due to gravity, subject to the end conditions

$$
y(0)=0, y\left(x_{1}\right)=y_{1} .
$$

Bernoulli, and those who responded successfully to his challenge, essentially derived and solved some form or consequence of the Euler-Lagrange equation for $T$. This EulerLagrange equation has the first integral

$$
y\left(1+y_{x}^{2}\right)=2 r
$$

where $r>0$ is a constant, from which the unique solution $\bar{y}$ of the Euler-Lagrange equation satisfying (2.1) can be obtained in parametric form for some $r$ and $\theta_{1} \in(0,2 \pi)$ as

$$
\begin{aligned}
& x(\theta)=r(\theta-\sin \theta), \\
& y(\theta)=r(1-\cos \theta),
\end{aligned}
$$

where $0 \leq \theta \leq \theta_{1}$. This solution represents the arc of a cycloid corresponding to the path traced out by a point $P$ starting at the origin and on the circumference of a circle of radius $r$ that rolls without slipping along the $x$-axis. The particle thus begins to fall vertically, rising as it approaches $\left(x_{1}, y_{1}\right)$ if and only if $y_{1} / x_{1}<2 / \pi$. However this analysis does not completely solve the problem since all that has been shown is that if there exists a (sufficiently smooth) minimizing curve then it is the cycloid. It is easy to forgive mathematicians of the time for this confusion between necessary and sufficient conditions, since they would not have been aware of one-dimensional problems for which the minimum is not attained. Here are two well-known such examples.

EXAMPLE 2.1. Consider the problem of minimizing the integral

$$
I(y)=\int_{0}^{1}\left[\left(y_{x}^{2}-1\right)^{2}+y^{2}\right] d x
$$

among functions $y$ that are at least absolutely continuous (so that in particular the derivative $y_{x}$ is defined almost everywhere) satisfying the end conditions

$$
y(0)=y(1)=0 .
$$

The infimum of $I$ among $y$ satisfying (2.3) is zero. In fact if $y^{(j)}$ is defined by

$$
y^{(j)}(x)=j^{-1} \theta(j x),
$$


where $\theta: \mathbf{R} \rightarrow \mathbf{R}$ is the 1-periodic function satisfying

$$
\theta(x)= \begin{cases}x, & \text { if } 0 \leq x \leq \frac{1}{2} \\ 1-x, & \text { if } \frac{1}{2}<x \leq 1\end{cases}
$$

then it is easily checked that $I\left(y^{(j)}\right) \rightarrow 0$ as $j \rightarrow \infty$. The $y^{(j)}$ are not smooth, but if desired we could easily smooth them (e.g. by suitably rounding corners) and still preserve the property $I\left(y^{(j)}\right) \rightarrow 0$.

However, there is no $y$ satisfying (2.3) with $I(y)=0$, since $y$ would have to satisfy the mutually incompatible conditions

$$
y=0, \quad\left|y_{x}\right|=1 .
$$

Hence $I$ does not attain a minimum subject to (2.3) in any usual function space.

A different point of view, pioneered by L. C. Young [113], is to generalize the concept of a function so that the conditions (2.4) are no longer incompatible. From this perspective $I$ attains a minimum at a generalized curve consisting of the track $y=0$ and associated Young measure $\left(\nu_{x}\right)$, corresponding to the sequence $z^{(j)}=y_{x}^{(j)}$ of derivatives of $y^{(j)}$, given by

$$
\nu_{x}=\frac{1}{2} \delta_{-1}+\frac{1}{2} \delta_{1}
$$

In general, the Young measure of a sequence of mappings $z^{(j)}: \Omega \rightarrow \mathbf{R}^{m}$, where $\Omega$ is an open subset of $\mathbf{R}^{n}$, is a probability measure on the range space $\mathbf{R}^{m}$ for almost all $x$, that gives the limiting distribution as $j \rightarrow \infty$ of the values of $z^{(j)}(x)$. Provided $z^{(j)}$ satisfies a mild bound (e.g. for $\Omega$ bounded provided $z^{(j)}$ is bounded in $L^{1}(\Omega)$ ) the existence of a Young measure for some subsequence of $z^{(j)}$ is guaranteed by standard results (see, for example, [3], [8], [109]). For this subsequence (which we do not relabel) we have that

$$
\nu_{x}=\lim _{r \rightarrow 0} \lim _{j \rightarrow \infty} \nu_{x, r}^{(j)}
$$

in the sense of weak* convergence of probability measures, where $\nu_{x, r}^{(j)}$ is the probability distribution of $z^{(j)}(\xi)$ as $\xi$ is chosen uniformly at random from the ball $B(x, r)$. The Young measure has the property that for any continuous function $f: \mathbf{R}^{m} \rightarrow \mathbf{R}$ the weak limit of $f\left(z^{(j)}\right)$ in $L^{1}(E)$ is given by $\left\langle\nu_{x}, f\right\rangle=\int_{\mathbf{R}^{m}} f(\tau) d \nu_{x}(\tau)$ for any compact subset $E$ of $\Omega$, whenever this limit exists.

In the example, $\nu_{x}$ is independent of $x$, and to obtain $(2.5)$ we note that since $z_{x}^{(j)}(x) \rightarrow$ $\{-1,1\}$ in measure, $\operatorname{supp} \nu_{x} \subset\{-1,1\}$ and so $\nu_{x}=\lambda(x) \delta_{-1}+(1-\lambda(x)) \delta_{1}$, where $0 \leq \lambda(x) \leq 1$. Since $z^{(j)} \rightarrow 0$ in $L^{2}(0,1)$ it follows that the weak limit $z_{x}$ of $z_{x}^{(j)}$ is zero. Thus $\int_{\mathbf{R}} \tau d \nu_{x}(\tau)=0$, implying that $\lambda(x)=\frac{1}{2}$ as required.

EXAMPLE 2.2. The second example (cf. Cesari [38, p. 7]) is that of minimizing

$$
I(y)=\int_{0}^{1} \sqrt{y^{2}+y_{x}^{2}} d x
$$

subject to the end conditions

$$
y(0)=0, \quad y(1)=1 .
$$


In this case the infimum of $I$ equals 1 and is not attained. In fact, for any absolutely continuous $y$ satisfying (2.7) we have

$$
I(y)>\int_{0}^{1} y_{x} d x=1
$$

while it is easily checked that if

$$
y^{(j)}(x)= \begin{cases}0, & \text { if } 0 \leq x \leq 1-\frac{1}{j} \\ j\left(x-1+\frac{1}{j}\right), & \text { if } 1-\frac{1}{j}<x \leq 1\end{cases}
$$

then $I\left(y^{(j)}\right) \rightarrow 1$.

The integrand in Example 2.2 is not so unlike that for the brachistochrone, and might give one pause for thought as to whether the minimum is attained in that case. However, it turns out that for the brachistochrone the minimum is attained, and is given by the unique arc $\bar{y}$ of the cycloid described above. A neat way of proving this, which we now sketch, is due independently to Kosmol [77] and Troutman [112].

We first make precise the space of admissible functions. Let

$$
\mathcal{A}=\left\{y \in A C\left[0, x_{1}\right]: y \geq 0, y(0)=0, y\left(x_{1}\right)=y_{1}\right\}
$$

Here $A C\left[0, x_{1}\right]$ denotes the space of absolutely continuous functions on the closed interval $\left[0, x_{1}\right] . A C\left[0, x_{1}\right]$ can be identified with the Sobolev space $W^{1,1}\left(0, x_{1}\right)$. We must show that $I(y)>I(\bar{y})$ if $y \in \mathcal{A}, y \neq \bar{y}$. Make the change of variables $z=\sqrt{2 y}$ to obtain the equivalent problem of minimizing

$$
I(z)=\int_{0}^{x_{1}} \sqrt{z^{-2}+z_{x}^{2}} d x
$$

in

$$
\overline{\mathcal{A}}=\left\{z \in A C\left[0, x_{1}\right]: z \geq 0, z(0)=0, z\left(x_{1}\right)=\sqrt{2 y_{1}}\right\} .
$$

To show that this really is an equivalent problem one checks that $y \in \mathcal{A}$ with $T(y)<\infty$ if and only if $z \in \overline{\mathcal{A}}$ with $I(z)<\infty$. Now note that the integrand $f(z, p)=\sqrt{z^{-2}+p^{2}}$ is a strictly convex function of $(z, p)$. Using the fact that a convex function lies above its tangent planes, we obtain

$$
I(z)-I(\bar{z})>\int_{0}^{x_{1}}\left[f_{z}\left(\bar{z}, \bar{z}_{x}\right)(z-\bar{z})+f_{p}\left(\bar{z}, \bar{z}_{x}\right)\left(z_{x}-\bar{z}_{x}\right)\right] d x
$$

if $z \neq \bar{z}$, where $\bar{z}=\sqrt{2 \bar{y}}$. Note that $\bar{z}(x)$ is a smooth solution of the Euler-Lagrange equation

$$
\frac{d}{d x} f_{p}=f_{z}
$$

corresponding to $I$, and that as $x \rightarrow 0+$ we have from (2.2) the estimates $\bar{z}(x) \sim$ $c_{1} x^{1 / 3}, \bar{z}_{x}(x) \sim \frac{1}{3} c_{1} x^{-2 / 3}$ for some constant $c_{1}=c_{1}(r)>0$. Hence, $f_{z}\left(\bar{z}, \bar{z}_{x}\right) \sim c_{2} x^{-1 / 3}$, 
$\left|f_{p}\left(\bar{z}, \bar{z}_{x}\right)\right| \leq 1$, and so

$$
\begin{aligned}
\int_{0}^{x_{1}}\left[f_{z}\left(\bar{z}, \bar{z}_{x}\right)(z-\bar{z})+f_{p}\left(\bar{z}, \bar{z}_{x}\right)\left(z_{x}-\bar{z}_{x}\right)\right] d & \\
& =\lim _{\varepsilon \rightarrow 0} \int_{\varepsilon}^{x_{1}} \frac{d}{d x}\left[f_{p}\left(\bar{z}, \bar{z}_{x}\right)(z-\bar{z})\right] d x=0,
\end{aligned}
$$

completing the proof that $\bar{y}$ is the unique minimizer. The reader is referred to Troutman's book for other examples of variational problems that can be convexified via a suitable change of variables.

For a different rigorous treatment of the brachistochrone problem from the point of view of optimal control theory, together with another attractive account of the history of the problem, see Sussmann and Willems [101]. Sussmann and Willems make the point that an advantage of the optimal control approach is that the form of the brachistochrone is proved to be a graph. This can be shown also using the calculus of variations (see Tonelli [110, Vol. II, pp. 405 ff.]).

3. Highlights of the one-dimensional theory. From a general perspective the brachistochrone problem is unusual, in that there is a unique solution of the EulerLagrange equation that is smooth on $\left(0, x_{1}\right]$ and is the unique absolute minimizer. In general there may be no absolute minimizer, or several local minimizers, and minimizers may or may not be smooth. In this section we examine some key results addressing these issues for general one-dimensional integrals on a finite interval $(a, b)$, having the form

$$
I(y)=\int_{a}^{b} f\left(x, y, y_{x}\right) d x
$$

with a corresponding set of admissible functions

$$
\mathcal{A}=\{y \in A C[a, b]: y(a)=\alpha, y(b)=\beta\},
$$

where $\alpha, \beta$ are given constants. Similar results can be given for more general boundary conditions. We suppose for simplicity that the integrand $f$ is smooth.

Note that if $y \in \mathcal{A}$ then $I(y)$ may be undefined as a Lebesgue integral, or be defined and finite, or be equal to $\pm \infty$.

3.1. The Weierstrass theory of local minimizers. Weierstrass realized that the theory of local minimizers for $I$ depends critically on the norm used. One such norm is that of the Sobolev space $W^{1, \infty}(a, b)$ of Lipschitz functions on $[a, b]$ and is given by

$$
\|v\|_{1, \infty}=\max \left(\|v\|_{\infty},\left\|v_{x}\right\|_{\infty}\right)
$$

with

$$
\|z\|_{\infty}=\|z\|_{L^{\infty}(a, b)}=\operatorname{ess~sup}_{x \in(a, b)}|z(x)| .
$$

We say that the function $\bar{y} \in \mathcal{A}$ is a local minimizer of $I$ in $W^{1, \infty}(a, b)$ (in the classical terminology a weak local minimizer) if there exists $\varepsilon>0$ such that whenever $y \in \mathcal{A}$ with $\|y-\bar{y}\|_{1, \infty}<\varepsilon$ then $I(y)$ is defined with $I(y) \geq I(\bar{y})$.

Denote by $C_{0}^{1}(a, b)$ the space of all $C^{1}$ functions $\varphi$ on $[a, b]$ with $\varphi(a)=\varphi(b)=0$. Necessary conditions for a function $\bar{y} \in C^{1}([a, b]) \cap \mathcal{A}$ to be a local minimizer of $I$ in 
$W^{1, \infty}(a, b)$ are the weak form of the Euler-Lagrange equation (or vanishing of the first variation)

$$
\delta I(\bar{y})(\varphi):=\left.\frac{d}{d \tau} I(\bar{y}+\tau \varphi)\right|_{\tau=0}=0, \quad \text { for all } \varphi \in C_{0}^{1}(a, b),
$$

and the positivity of the second variation

$$
\delta^{2} I(\bar{y})(\varphi):=\left.\frac{d^{2}}{d \tau^{2}} I(\bar{y}+\tau \varphi)\right|_{\tau=0} \geq 0, \quad \text { for all } \varphi \in C_{0}^{1}(a, b) .
$$

We abbreviate the necessary conditions (3.1) and (3.2) by

$$
\delta I(\bar{y})=0
$$

and

$$
\delta^{2} I(\bar{y}) \geq 0,
$$

respectively. A standard argument based on a Taylor expansion shows that if we strengthen (3.4) to

$$
\delta^{2} I(\bar{y})>0
$$

that is, for some constant $\mu>0$

$$
\left.\frac{d^{2}}{d \tau^{2}} I(\bar{y}+\tau \varphi)\right|_{\tau=0} \geq \mu \int_{a}^{b}\left(\varphi^{2}+\varphi_{x}^{2}\right) d x, \quad \text { for all } \varphi \in C_{0}^{1}(a, b),
$$

then (3.3), (3.5) are together sufficient for a function $\bar{y} \in C^{1}([a, b]) \cap \mathcal{A}$ to be a local minimizer of $I$ in $W^{1, \infty}(a, b)$.

Now suppose that $\bar{y} \in C^{1}([a, b]) \cap \mathcal{A}$ is a local minimizer in $L^{\infty}(a, b)$, that is, there exists $\varepsilon>0$ such that if $y \in \mathcal{A}$ with $\|y-\bar{y}\|_{\infty}<\varepsilon$ then $I(y)$ is defined with $I(y) \geq I(\bar{y})$. Then $\bar{y}$ satisfies the Weierstrass condition, that is, for every $x \in[a, b]$ the tangent to the graph of $p \mapsto f(x, \bar{y}(x), p)$ at $p=\bar{y}_{x}$ does not lie above the graph. The Fundamental Sufficiency Theorem of Weierstrass states that adding an appropriately strengthened version of the Weierstrass condition gives a set of sufficient conditions for $\bar{y}$ to be a local minimizer in $L^{\infty}(a, b)$. The strengthened Weierstrass condition is that there exists an $\varepsilon>0$ such that given any $x \in[a, b]$, and any $v, q$ with $|v-\bar{y}(x)|<\varepsilon,\left|q-\bar{y}_{x}\right|<\varepsilon$, the tangent to the graph of $p \mapsto f(x, v, p)$ at $p=q$ does not lie above this graph. A modern statement of the theorem is (see Hestenes [67]):

TheOrEm 3.1 (Fundamental Sufficiency Theorem). Let $\bar{y} \in C^{1}([a, b]) \cap \mathcal{A}$ satisfy the three conditions:

(i) $\delta I(\bar{y})=0$,

(ii) $\delta^{2} I(\bar{y})>0$,

(iii) the strengthened Weierstrass condition.

Then $\bar{y}$ is a local minimizer of $I$ in $L^{\infty}(a, b)$. In fact, there exist constants $\sigma>0$, $\varepsilon>0$ such that

$$
I(y) \geq I(\bar{y})+\sigma \int_{a}^{b} D\left(y_{x}-\bar{y}_{x}\right) d x
$$

whenever $\|y-\bar{y}\|_{\infty}<\varepsilon$, where $D(t):=\sqrt{1+t^{2}}-1$. 
The classical proof of Theorem 3.1 is via the field theory of the calculus of variations, as introduced by Weierstrass and simplified using null Lagrangians by Hilbert. It is this proof that appears in almost all texts. A second "indirect sufficiency proof" due to Hestenes can be found in his book [67], and has the advantage that it applies in situations (for example, problems with isoperimetric constraints) for which the field theory does not, or is difficult to, apply. A direct version of the Hestenes proof based on finite elements is due to R. D. James and the author [18]. For yet another proof see Clarke and Zeidan [41].

3.2. Tonelli theory. During the years 1918-1939 Leonidas Tonelli made a systematic study of the one-dimensional calculus of variations, and in particular of weak lower semicontinuity, the key to the direct method of the calculus of variations. In his work he could take advantage of the newly-developed Lebesgue integral. He proved the following fundamental existence theorem.

TheOREm 3.2 (Tonelli [110, II p. 282], [111]). Suppose that $f(x, y, p)$ is convex in $p$ for each $x \in[a, b], y \in \mathbf{R}$, and that $f$ satisfies the growth condition

$$
f(x, y, p) \geq \Phi(p)
$$

for some function $\Phi$ that is superlinear, i.e.,

$$
\lim _{|p| \rightarrow \infty} \frac{\Phi(p)}{|p|}=\infty
$$

Then $I$ attains an absolute minimum on $\mathcal{A}$.

Note that in Example 2.1, $f(y, p)$ is not convex in $p$ but satisfies the growth condition (3.8), while in Example 2.2, $f(y, p)$ is convex in $p$ but does not satisfy (3.8). For the brachistochrone problem (3.8) is again not satisfied. However, there is a modification of Tonelli's existence theorem that does apply to the brachistochrone example (see Cesari $[38$, p. 439]).

Much less well known, indeed apparently forgotten for half a century, is Tonelli's partial regularity theorem, the forerunner of modern partial regularity theorems for multiple integrals.

Theorem 3.3 (Tonelli [110, II p. 359]). Let $f_{p p}(x, y, p)>0$ for all $x \in[a, b], y, p$ and let $\bar{y}$ be a local minimizer of $I$ in $L^{\infty}(a, b)$. Then $\bar{y} \in C^{\infty}([a, b] \backslash E)$, where $E$ is closed and $\mathcal{L}^{1}(E)=0$. Furthermore, the derivative

$$
\bar{y}_{x}(x):=\lim _{h \rightarrow 0} \frac{y(x+h)-y(x)}{h}
$$

(one-sided if $x=a$ or $x=b$ ) exists for all $x \in[a, b]$ and is a continuous function from $[a, b]$ to the extended real line $\overline{\mathbf{R}}$, with $\left|\bar{y}_{x}(x)\right|=\infty$ for $x \in E$.

Here and below $\mathcal{L}^{n}$ denotes $n$-dimensional Lebesgue measure. The assertion that $\bar{y}_{x}:[a, b] \rightarrow \overline{\mathbf{R}}$ is continuous is due to Ball and Mizel [26]. Tonelli did not know of an example in which the Tonelli set $E$ was nonempty, and examples were first provided in [25], [26]. 
EXAMPLE $3.1([25],[26])$. Consider the problem of minimizing the integral

$$
I(y)=\int_{-1}^{1}\left[\left(x^{4}-y^{6}\right)^{2} y_{x}^{28}+\varepsilon y_{x}^{2}\right] d x
$$

in the set

$$
\mathcal{A}=\left\{y \in W^{1,1}(-1,1): y(1)=1, y(-1)=-1\right\},
$$

where $0<\varepsilon<<1$. Provided $\varepsilon$ is sufficiently small, any minimizer $\bar{y}$ of $I$ in $\mathcal{A}$ is smooth on $[-1,0) \cup(0,1]$, but $\bar{y}(x) \sim|x|^{2 / 3} \operatorname{sgn} x, \bar{y}_{x}(x) \sim \frac{2}{3}|x|^{-1 / 3}$ as $x \rightarrow 0$, so that the Tonelli set $E=\{0\}$. Thus the singularity in the minimizer $y=|x|^{2 / 3} \operatorname{sgn} x$ for $\varepsilon=0$ is not destroyed when $\varepsilon$ is positive and sufficiently small, even though the Euler-Lagrange equation for (3.9) is an elliptic ordinary differential equation of the form $y_{x x}=F_{\varepsilon}\left(x, y, y_{x}\right)$ with $F_{\varepsilon}$ smooth. This equation fails to smooth the singularity because $\bar{y}$ does not satisfy the weak form of it on the whole of $[-1,1]$. In this example, though not in others in which $E$ is nonempty, the nonemptiness of $E$ is related to the remarkable Lavrentiev phenomenon (see Lavrentiev [79], Manià [83]), that

$$
\inf _{y \in \mathcal{A} \cap W^{1, \infty}(-1,1)} I(y)>\inf _{y \in \mathcal{A}} I(y)=I(\bar{y}) .
$$

In fact, in this example, the minimum of $I$ in $\mathcal{A} \cap W^{1, \infty}(-1,1)$ is attained by a pair of smooth functions $\pm y^{*}$ that do not pass through the origin. A further remarkable property that holds is that $I\left(y^{(j)}\right) \rightarrow \infty$ for any sequence of smooth functions $y^{(j)}$ satisfying $y^{(j)}(x) \rightarrow \bar{y}(x)$ almost everywhere. These properties have the implication that natural finite-element schemes based on piecewise-affine approximations will fail to detect $\bar{y}$ and instead converge to $\pm y^{*}$. Various more subtle numerical schemes (see Ball and Knowles [23], Li [80], [81]) succeed in detecting singular minimizers.

Note the surprising fact that it pays overall to have the integrand infinite somewhere (so that the minimizer can stay closer to $|x|^{2 / 3} \operatorname{sgn} x$ elsewhere). Some idea as to why the Euler-Lagrange equation is not satisfied on $[a, b]$ can be obtained from noting (see [26]) that $I(\bar{y}+\tau \varphi)=\infty$ unless $\tau=0$ for any smooth $\varphi$ with $\varphi(0) \neq 0$. In Davie [48] (see also [26] for the case $f=f(y, p)$ ) it is shown that the theorem is optimal, in the sense that given any closed set $E \subset[a, b]$ there exists a corresponding $f$ satisfying the hypotheses of the theorem, and boundary values $\alpha, \beta$, such that the Tonelli set equals $E$. For further results and references on singular minimizers and the Lavrentiev phenomenon, see Ball and Nadirashvili [28], Sychev [105], [106], [107].

4. Multiple integral problems. Consider the integral

$$
I(y)=\int_{\Omega} f(x, y(x), D y(x)) d x
$$

where $\Omega \subset \mathbf{R}^{n}$ is bounded and open with Lipschitz boundary $\partial \Omega$, and $y: \Omega \rightarrow \mathbf{R}^{m}$. Here

$$
D y(x)=\left(\frac{\partial y_{i}}{\partial x_{j}}(x)\right) \in M^{m \times n}
$$

denotes the derivative of $y$ at $x$, where $M^{m \times n}$ denotes the space of real $m \times n$ matrices. We assume for simplicity that $f: \Omega \times \mathbf{R}^{m} \times M^{m \times n} \rightarrow \mathbf{R}$ is smooth. Our aim is to study 
minimizers of $I$ in the set of admissible mappings

$$
\mathcal{A}=\left\{y \in W^{1,1}\left(\Omega ; \mathbf{R}^{m}\right):\left.y\right|_{\partial \Omega_{1}}=g\right\},
$$

where $\partial \Omega_{1}$ is a subset of $\partial \Omega$ with positive $(n-1)$-dimensional Hausdorff measure $\mathcal{H}^{n-1}\left(\partial \Omega_{1}\right)$ and $g$ is a given measurable mapping that is the trace of some $y \in W^{1,1}(\Omega$; $\mathbf{R}^{m}$ ) (so that $\mathcal{A}$ is nonempty). We have here chosen a typical set of mixed boundary conditions, in which the remaining part $\partial \Omega_{2}=\partial \Omega \backslash \partial \Omega_{1}$ of the boundary is free. Because we do not specify $y$ there we expect minimizers to satisfy the natural boundary condition

$$
D_{A} f \cdot N(x)=0, \quad x \in \partial \Omega_{2},
$$

where $D_{A} f$ denotes the derivative of $f(x, y, A)$ with respect to the matrix $A$, and $N(x)$ denotes the unit outward normal to $\partial \Omega$.

A motivating example is that of nonlinear elastostatics, when $m=n=3$ and

$$
I(y)=\int_{\Omega} \psi(D y) d x
$$

where $\psi=\psi_{\theta}$ denotes the free-energy function of an elastic material (assumed homogeneous) at a fixed temperature $\theta$. However, in this case $\psi=\psi(A)$ is not smooth, but is typically assumed to be defined only for $A \in M^{3 \times 3}$ with $\operatorname{det} A>0$, and to satisfy the condition

$$
\psi(A) \rightarrow \infty \quad \text { as } \operatorname{det} A \rightarrow 0+,
$$

corresponding to the requirement that infinite energy is needed to compress the material to zero volume. This introduces extra technical difficulties that we do not treat here (see [5], [6], V. Šverák [102]).

C. B. Morrey [87] discovered the correct generalization of convexity of $f(x, y, \cdot)$ for multiple integral problems. Consider the case when $f=f(A)$, i.e., $f: M^{m \times n} \rightarrow \mathbf{R}$.

Definition 4.1. If $A \in M^{m \times n}$ then $f$ is quasiconvex at $A$ if

$$
\int_{\Omega} f(D v(x)) d x \geq \int_{\Omega} f(A) d x=\mathcal{L}^{n}(\Omega) f(A)
$$

for all $v \in W^{1, \infty}\left(\Omega ; \mathbf{R}^{m}\right)$ satisfying the linear boundary condition $v(x)=A x$ for all $x \in \partial \Omega$. We say that $f$ is quasiconvex if it is quasiconvex at every $A \in M^{m \times n}$.

Despite first appearances this definition is independent of $\Omega$. If either $m=1$ or $n=1$ quasiconvexity of $f$ is the same as convexity. However, this is definitely not the case for $m>1$ and $n>1$, and this makes the multi-dimensional calculus of variations an altogether more difficult subject.

There are important relations between quasiconvexity and certain other convexity conditions. We say that $f$ is polyconvex if $f(A)=g(\mathbf{J}(A))$ for some convex function $g$, where $\mathbf{J}(A)$ denotes the vector consisting of all minors (i.e. subdeterminants) of $A$. Thus, for example, if $m=n=3, f$ is polyconvex if and only if there is a convex function $g: M^{3 \times 3} \times M^{3 \times 3} \times \mathbf{R} \rightarrow \mathbf{R}$ with

$$
f(A)=g(A, \operatorname{cof} A, \operatorname{det} A) \quad \text { for all } A \in M^{3 \times 3},
$$

where cof $A$ denotes the matrix of cofactors of $A$. We say that $f$ is rank-one convex if

$$
\frac{d^{2}}{d \tau^{2}} f(A+\tau \lambda \otimes \mu) \geq 0
$$


for all nonzero $\lambda \in \mathbf{R}^{m}, \mu \in \mathbf{R}^{n}$. The corresponding condition with strict inequality in (4.3) is known as strong ellipticity.

It is not hard to establish the implications (see, for example, [5], Dacorogna [45])

$$
f \text { polyconvex } \Rightarrow f \text { quasiconvex } \Rightarrow f \text { rank-one convex. }
$$

Examples of quasiconvex functions that are not polyconvex are known for all dimensions $m \geq 2, n \geq 2$ (see Zhang [117]). We discuss the reverse implication of whether rank-one convexity implies quasi-convexity in Section 6 .

Morrey showed that $f(x, y, \cdot)$ quasiconvex is necessary and sufficient (under appropriate growth hypotheses) for $I$ to be sequentially weakly lower semicontinuous on $W^{1, p}=W^{1, p}\left(\Omega ; \mathbf{R}^{m}\right)$, where $p>1$, i.e.,

$$
I(y) \leq \liminf _{j \rightarrow \infty} I\left(y^{(j)}\right)
$$

whenever $y^{(j)} \rightarrow y$ in $W^{1, p}$. Later work of Acerbi and Fusco [2] weakened Morrey's growth hypotheses to show that (4.4) holds if

$$
0 \leq f(x, y, A) \leq C\left(1+|A|^{p}\right) \quad \text { for all } x, y, A,
$$

and some constant $C$ (even if $f$ is only measurable in $x$ and continuous in $(y, A)$ ). Hence one can prove the existence of an absolute minimizer for $I$ in $\mathcal{A}$, provided $f$ is also coercive, i.e.,

$$
f(x, y, A) \geq C_{0}|A|^{p}-1 \quad \text { for all } x, y, A,
$$

and some constant $C_{0}>0$. Quasiconvexity is in a sense also necessary for the existence of minimizers. For example, as was shown in [27], for $f$ of the form

$$
f(x, y, A)=W(A)+\psi(x, y)
$$

if $I$ attains an absolute minimum for all linear boundary data $\left.y\right|_{\partial \Omega}=C x$ and all continuous nonnegative $\psi$, then $W$ is quasiconvex.

Quasiconvexity plays other central roles in the multi-dimensional calculus of variations. For example, there is a key partial regularity theorem of Evans [59] which asserts that under suitable growth conditions and an appropriate strengthening of quasiconvexity, any absolute minimizer $y$ of $I$ in $\mathcal{A}$ is smooth off a closed subset $E \subset \Omega$ with $\mathcal{L}^{n}(E)=0$.

Another key result is the relaxation theorem of Dacorogna [44], [45], which concerns the quasiconvexification $Q f$ with respect to the gradient of an integrand $f$ such that $f(x, y, \cdot)$ is not quasiconvex (so that minimizers may not exist), defined by

$$
Q f(x, y, A)=\sup \{g(A): g \text { quasiconvex with } g(B) \leq f(x, y, B) \text { for all } B\} .
$$

The theorem states that if $f$ satisfies suitable growth conditions then the functional

$$
Q I(y)=\int_{\Omega} Q f(x, y, D y) d x
$$

characterizes the weak limits of minimizing sequences for $I$ on $\mathcal{A}$. More precisely, inf $\mathcal{A}_{\mathcal{A}} I=$ $\inf _{\mathcal{A}} Q I$, the weak limit $y$ in $W^{1, p}$ of any minimizing sequence $y^{(j)}$ for $I$ in $\mathcal{A}$ is a minimizer for $Q I$ in $\mathcal{A}$, and any minimizer $y$ for $Q I$ in $\mathcal{A}$ is the $W^{1, p}$ weak limit of some minimizing sequence for $I$. 
There is also a remarkable connection between gradient Young measures and quasiconvexity due to Kinderlehrer and Pedregal [72]. A family $\left(\nu_{x}\right)$ of probability measures on $M^{m \times n}$ depending measurably on $x \in \Omega$ is the Young measure of a sequence $D y^{(j)}$ of gradients bounded in $L^{\infty}$ if and only if (i) the centre of mass $\bar{\nu}_{x}$ is a gradient, and (ii) Jensen's inequality

$$
\left\langle\nu_{x}, f\right\rangle \geq f\left(\bar{\nu}_{x}\right)
$$

holds for a.e. $x$ and all quasiconvex $f$. There is also an $L^{p}$ version of this result (Kinderlehrer and Pedregal [73]).

One can also study quasiconvex sets. A compact set $E \subset M^{m \times n}$ is quasiconvex if it is the zero-set of a nonnegative quasiconvex function. The quasiconvexification $Q(E)$ of a set $E$ is the intersection of all quasiconvex sets containing $E$. As we will see in Section 5 , these sets are of interest in connection with microstructure in materials. As an example of a developing theory of quasiconvex sets, Zhang [116] has proved the following interesting generalization of the finite-dimensional Krein-Milman theorem; if $E \subset M^{m \times n}$ is compact and quasiconvex, then it is the quasiconvexification of the set of its quasiconvex extreme points. Here a quasiconvex extreme point $A \in E$ is one such that if $\nu$ is a gradient Young measure supported in $E$ and with centre of mass $\bar{\nu}=A$ then $\nu=\delta_{A}$. For other results see Zhang [115].

5. The elasticity model of martensitic transformations. Most alloys undergo solid phase transformations involving a change of shape at one or more critical temperatures. The calculus of variations gives insight into why such phase transformations typically lead to patterns of fine microstructure, in the simplest case consisting of a laminate of parallel thin layers in which the spatial gradient of the deformation alternates between two values. Roughly, the idea (cf. Ball and James [20]) is that according to a model based on nonlinear elastostatics, the minimum of the corresponding energy functional is not in general attained, so that the fine microstructure observed can be thought of as an element of a minimizing sequence that oscillates more and more finely. At macroscopic length-scales the deformation gradient can be smooth, even if at the microscopic scale it oscillates wildly. The analysis of the elasticity model of microstructure has provided a much needed stimulus to the multi-dimensional calculus of variations, posing fundamental questions concerning quasiconvexity, relaxation, and gradient Young measures.

Consider a single crystal of such an alloy that occupies the bounded open subset $\Omega \subset \mathbf{R}^{3}$ in a reference configuration. A typical deformation of the crystal is described by the mapping $y: \Omega \rightarrow \mathbf{R}^{3}$, where $y(x)$ is the deformed position of the material point with position $x$ in the reference configuration. The elastic energy of the crystal is given by the integral

$$
I_{\theta}(y)=\int_{\Omega} \psi_{\theta}(D y(x)) d x
$$

where $\psi_{\theta}$ is the free-energy function of the material and $\theta$ is the temperature. In this model we ignore other possible contributions to the energy, such as any energy associated to interfaces across which $D y$ jumps. The temperature is assumed to be constant. 
We assume that the minimum value of $\psi_{\theta}(A)$ among matrices $A \in M^{3 \times 3}$ is zero. Provided $\psi_{\theta}$ is bounded below this can be arranged by adding to it a suitable function of $\theta$. We focus attention on the values of $A$ that minimize $\psi_{\theta}(A)$, that is, on the set $K=K(\theta)$ given by

$$
K(\theta)=\left\{A \in M^{3 \times 3}: \psi_{\theta}(A)=0\right\} .
$$

Because of the frame-indifference condition

$$
\psi_{\theta}(R A)=\psi_{\theta}(A) \quad \text { for all } A \in M^{3 \times 3}, R \in S O(3),
$$

this set typically has the form

$$
K(\theta)=\bigcup_{i=1}^{N} S O(3) V_{i}
$$

where $N=N(\theta)$ and $V_{i}=V_{i}(\theta)=V_{i}^{\mathrm{T}}>0$. For example, in a cubic to tetragonal transformation with critical temperature $\theta_{c}$ and lattice parameters $\eta_{1}>0, \eta_{2}>0$, we have

$$
K\left(\theta_{c}\right)=S O(3) \cup \bigcup_{i=1}^{3} S O(3) U_{i}
$$

where

$$
\begin{aligned}
& U_{1}=\operatorname{diag}\left(\eta_{2}, \eta_{1}, \eta_{1}\right), \\
& U_{2}=\operatorname{diag}\left(\eta_{1}, \eta_{2}, \eta_{1}\right), \\
& U_{3}=\operatorname{diag}\left(\eta_{1}, \eta_{1}, \eta_{2}\right) .
\end{aligned}
$$

Thus there are four energy wells, $S O(3)$ corresponding to the undistorted cubic phase (which we take as reference configuration), and the three others to variants of the tetragonal phase in which the cubic elementary cell of the crystal lattice changes shape to a tetragon with two sides equal to $\eta_{1}$ and the third of length $\eta_{2}$. For temperatures $\theta>\theta_{c}$,

$$
K(\theta)=k(\theta) S O(3),
$$

where $k(\theta)$ measures the thermal expansion, with $k\left(\theta_{c}\right)=1$, while for $\theta<\theta_{c}$,

$$
K(\theta)=\bigcup_{i=1}^{3} S O(3) U_{i}
$$

with the $U_{i}$ as in (5.1) but with the $\eta_{i}=\eta_{i}(\theta)$.

To see how we can describe microstructures in this framework, consider the case of a simple laminate defined by a mapping $y^{(j)}$, whose gradient takes the values $A, B \in M^{3 \times 3}$ in alternate layers separated by planar interfaces with normal $n$. For this to be possible we must have

$$
A-B=a \otimes n
$$

for some $a \in \mathbf{R}^{3}$. We suppose that the layers in which $D y^{(j)}=A$ have thickness $\lambda / j$, and that the layers in which $D y^{(j)}=B$ have thickness $(1-\lambda) / j$, where $0<\lambda<1$. If we let $j \rightarrow \infty$ we get an infinitely fine laminate. The Young measure $\left(\nu_{x}\right)$ corresponding to the sequence of gradients $D y^{(j)}$ can be identified from the probabilistic description (2.6). 
Clearly one has a chance $\lambda$ of finding the value $D y^{(j)}=A$ and $1-\lambda$ of finding the value $D y^{(j)}=B$, and hence $\nu_{x}=\nu$ is independent of $x$ and given by

$$
\nu=\lambda \delta_{A}+(1-\lambda) \delta_{B}
$$

The corresponding macroscopic deformation gradient is the weak limit $D y$ of $D y^{(j)}$, namely

$$
D y=\lambda A+(1-\lambda) B
$$

In general, the passage from the microscopic continuum theory to a macroscopic one is achieved via weak limits and quasiconvexification. For example, according to the relaxation theorem of Dacorogna (see Section 4) we expect the macroscopic deformation (weak limit) $y$ to minimize the relaxed integral

$$
Q I(y)=\int_{\Omega} Q \psi(D y) d x
$$

where we have suppressed the dependence on $\theta$. (See, however, Section 6.1.) A special case is that of microstructures of least possible energy, corresponding to sequences $D y^{(j)}$ of deformation gradients with $D y^{(j)}$ tending in measure to the set of energy wells $K$. The associated Young measures $\left(\nu_{x}\right)$ are those with

$$
\operatorname{supp} \nu_{x} \subset K \quad \text { a.e. } x \in \Omega \text {. }
$$

The corresponding macroscopic deformation gradients are those with values in the quasiconvexification $Q(K)$, which can be expressed in the alternative forms

$$
\begin{aligned}
Q(K) & =\left\{A \in M^{3 \times 3}: \inf _{y \mid \partial \Omega=A x} \int_{\Omega} \psi(D y) d x=0\right\} \\
& =\{\bar{\nu}: \nu \text { a homogeneous gradient Young measure with supp } \nu \subset K\} .
\end{aligned}
$$

Unfortunately the computation of $Q(K)$ is a nontrivial task. For example, it is an open problem to calculate $Q(K)$ when $K$ consists of the three tetragonal wells in a cubic-totetragonal transformation, i.e. when

$$
K=\bigcup_{i=1}^{3} S O(3) U_{i}
$$

However, the computation can be done for the case of just two tetragonal wells, namely for

$$
K=S O(3) U_{1} \cup S O(3) U_{2}
$$

to which the general case

$$
K=S O(3) U \cup S O(3) V
$$

of two wells with $\operatorname{det} U=\operatorname{det} V$ that are rank-one connected (i.e. $\operatorname{rank}(U-R V)=1$ for some $R \in S O(3)$ ) can be reduced via a linear change of variables (cf. Ball and James $[22])$. 
Theorem 5.1 (Ball and James [22], [21]). For $K$ given by $(5.3), Q(K)$ consists of those $A \in M^{3 \times 3}$ with $\operatorname{det} A>0$ such that

$$
A^{\mathrm{T}} A=\left(\begin{array}{ccc}
a & c & 0 \\
c & b & 0 \\
0 & 0 & \eta_{1}^{2}
\end{array}\right),
$$

where $a>0, b>0$, and

$$
\begin{aligned}
a+b \pm 2 c & \leq \eta_{1}^{2}+\eta_{2}^{2}, \\
a b-c^{2} & =\eta_{1}^{2} \eta_{2}^{2} .
\end{aligned}
$$

Furthermore, any invertible $y$ with $D y(x) \in Q(K)$ a.e. is a plane strain, i.e.

$$
y(x)=Q\left(z_{1}(x), z_{2}(x), \eta_{1} x_{3}+\mu\right)
$$

with $Q \in S O(3)$, and

$$
\frac{\partial z_{1}}{\partial x_{3}}=\frac{\partial z_{2}}{\partial x_{3}}=0
$$

The proof is in part based on the minors relations

$$
<\nu_{x}, J>=J\left(\bar{\nu}_{x}\right),
$$

for any minor $J=J(A)$ of $A$. These are the relations obtained by considering the case when equality holds in (4.5). Clearly equality holds if and only if $\pm f$ is quasiconvex, which is known to be true (see [5], Dacorogna [45]) if and only if $f$ is an affine combination of minors. It is apparently a piece of luck that one can get away with using just these relations rather than the general set (4.5).

As observed by Ball and Carstensen [11], the plane strain assertion in Theorem 5.1 leads immediately to a statement concerning non-attainment.

Corollary 5.2. If $A \in Q(K) \backslash K$ then

$$
\inf _{y \mid \partial \Omega=A x} \int_{\Omega} \psi(D y) d x
$$

is not attained.

Proof. Suppose $A \in Q(K)$ and that $y$ is a minimizer. Then $D y(x) \in K$ a.e.. Since $\left.y\right|_{\partial \Omega}$ is linear, a known invertibility theorem [6] ensures that $y$ is invertible. Hence by the theorem $y$ is a plane strain. But it is easily shown that a plane strain that is linear on the boundary of a (three-dimensional) region must itself be linear. Hence $D y=A$ is constant, and so $\psi(A)=0$, i.e. $A \in K$.

It follows from Corollary 5.2 that for $A \in Q(K) \backslash K$ minimizing sequences for $I$ generate microstructure. In two dimensions the corresponding result is false (cf. Müller and Šverák [90]).

It is not possible to do justice here to the many interesting contributions to this area; so instead we give an incomplete set of additional references for various topics:

General surveys. Ball and James [17], Luskin [82], Müller [89], Pitteri and Zanzotto [99]. Continuum theories of crystals and twinning. Ericksen [54], [55], [56], [57], [58], Gurtin [64], James [68], Pitteri [98], Zanzotto [114]. 
Microstructures and energy-wells. Bhattacharya [32], Bhattacharya, Firoozye, James, and Kohn [33], Chipot and Kinderlehrer [39], Fonseca [60], Hane [65], Kinderlehrer [71], Matos [84], Šverák [104]. For the special case of austenite-martensite interfaces see [20] and Ball and Carstensen [11], [12]. For special microstructures, including those that are only possible when special relations between lattice parameters hold, see Bhattacharya [30], [31], Ruddock [100]. For interesting explicit examples of quasiconvexifications see Kohn [74], Pipkin [96], [97].

Metastability and hysteresis. Abeyaratne, Chu and James [1], Ball, Chu and James [13], [14], Ball and James [19], Chu [40].

Theories with interfacial energy. Fonseca [61], Dolzmann and Müller [52], Kohn and Müller [75], [76].

Polycrystals. Bhattacharya and Kohn [34], [35].

Numerical computations. Carstensen and Plecháč [37], [36], Collins, Kinderlehrer and Luskin [42], Collins and Luskin [43], Dolzmann [51].

Magneto-elastic materials. A. DeSimone [50], James and Kinderlehrer [69], [70].

Dynamics and minimizing sequences. Ball [9], [10], Ball, Holmes, James, Pego and Swart [16], Friesecke and McLeod [62], Pego [95].

6. Some outstanding open problems. In this section I highlight some important open problems of the multi-dimensional theory in the context (see Section 4) of integrals of the form

$$
I(y)=\int_{\Omega} f(x, y, D y) d x
$$

subject to mixed boundary conditions

$$
\left.y\right|_{\partial \Omega}=g,
$$

where the hypotheses are as for (4.1).

6.1. To characterize quasiconvex functions. The problem is to give a tractable characterization of quasiconvex functions. At present we do not know how to verify whether or not a given $f=f(D y)$ is quasiconvex. Morrey introduced quasiconvexity in his 1952 paper [87]. Later, in his 1966 book [88] he wrote (with a change to modern terminology), "It is an unsolved problem to prove or disprove the theorem that every rank-one convex function is quasiconvex". This conjecture was finally disproved in 1992 by Šverák [103], who for the dimensions $n \geq 2, m \geq 3$ found a quartic polynomial that was rank-one convex but not quasiconvex. This eliminated the only existing plausible conjecture for a characterization. (For a review of the question prior to Šverák's work see [7].) The cases $n \geq 2, m=2$ remain open, and there is some circumstantial evidence that rank-one convexity could be the same as quasiconvexity if $m=n=2$ (see Dacorogna and Haeberly [46], [47], Parry [92], Pedregal [93], Pedregal and Šverák [94]).

In his original paper [87] Morrey had written: "In fact, after a great deal of experimentation, the writer is inclined to think that there is no condition of the type discussed, 
which involves $f$ and only a finite number of its derivatives, and which is both necessary and sufficient for quasiconvexity in the general case". Morrey's intuition was vindicated when Kristensen [78] used Šverák's example to show that for $n \geq 2, m \geq 3$ there is no local condition on $f$ that is necessary and sufficient for $f$ to be quasiconvex. Thus, for these dimensions, any characterization must be nonlocal (e.g. of the form that a function is quasiconvex if it is the supremum of some family of special quasiconvex functions).

A by-product of a successful characterization might be to make the theory of quasiconvexity apply to elasticity. At present the basic existence, regularity, and relaxation theorems described in Section 4 do not apply to elasticity because they make growth hypotheses that are inconsistent with the property (4.2). It is hard to see how to extend the current proofs without a better understanding of approximation by piecewise-affine functions in the presence of pointwise constraints on the determinant. However, a new characterization of quasiconvexity might lead to different proof ideas for which these approximation problems could disappear.

The problem of characterizing quasiconvexity can be posed also for integrals depending on higher derivatives (cf. Meyers [86], Ball, Currie and Olver [15]). In this context the problem is open even in the scalar case. For example, there are no known tractable conditions on $f$ that are necessary and sufficient for the integral

$$
I(u)=\int_{\Omega} f\left(u_{x x}, u_{y y}, u_{x y}\right) d x
$$

to be sequentially weak* lower semicontinuous in $W^{2, \infty}(\Omega)$, where $\Omega \subset \mathbf{R}^{2}$ is bounded, open, and $u: \Omega \rightarrow \mathbf{R}$.

6.2. To extend the Weierstrass theory of local minimizers to multiple integrals. The goal here is to find a set of necessary conditions for a given $\bar{y}$ to be a local minimizer of $I$ with respect to the boundary conditions $(6.2)$ in the space $W^{1, p}\left(\Omega ; \mathbf{R}^{m}\right), 1 \leq p<\infty$, which when slightly strengthened will also be sufficient. The natural generalizations of the Weierstrass condition to the case of multiple integrals with $m>1, n>1$ are the conditions of quasiconvexity in the interior, namely that if $x \in \Omega$ then $f(x, \bar{y}(x), \cdot)$ is quasiconvex at $D \bar{y}(x)$, and of quasiconvexity at the boundary, which is a similar condition applying to boundary points $x \in \partial \Omega_{2}=\partial \Omega \backslash \partial \Omega_{1}$ (see Ball and Marsden [24]). So a conjecture would be that if $\bar{y}$ is sufficiently smooth and satisfies the weak form of the Euler-Lagrange equation, the strict positivity of the second variation (the natural generalization of (3.6)), and appropriate generalizations of quasiconvexity in the interior and at the boundary, then $\bar{y}$ is a local minimizer of $I$ in $W^{1, p}$. The methods of field theory seem doomed to fail, since they rely on null Lagrangians (integrands whose EulerLagrange equations are identically satisfied), and these are associated with polyconvexity rather than quasiconvexity. When $m=1$ or $n=1$ the quasiconvexity conditions reduce to the Weierstrass condition, and the Fundamental Sufficiency Theorem holds. For the case $n=1, m>1$ see, for example, Hestenes [67]. The case $n>1, m=1$ is less well known; see Hestenes [66] and, for a modern treatment and other results, Taheri [108].

6.3. Regularity of minimizers. Since the work of De Giorgi [49] and Giusti and Miranda [63] it has been well known that minimizers of "elliptic" multiple integral problems can be singular. Most examples of such singular minimizers are discontinuous; for example, of the form $y(x)=x /|x|$. An exception is the example of Nečas [91], who constructed 
an example of a strictly convex smooth integrand $f=f(D y)$ such that

$$
I(y)=\int_{B} f(D y) d x
$$

where $B \subset \mathbf{R}^{n}$ is a ball and $y: B \rightarrow \mathbf{R}^{n^{2}}, n \geq 5$, with unique minimizer

$$
\bar{y}_{i j}(x)=\frac{x_{i} x_{j}}{|x|}
$$

subject to its own boundary conditions. Note that $\bar{y}$ is Lipschitz but not $C^{1}$.

Two important open questions are:

(1) Are there examples of strictly quasiconvex integrands $f=f(D y)$ such that $I$ has a singular minimizer $\bar{y}$ that is continuous but whose derivative $D \bar{y}$ is unbounded in the interior? The condition that $\bar{y}$ is continuous is automatic by the Sobolev embedding theorem provided $f$ satisfies the growth condition

$$
f(A) \geq C_{0}|A|^{p}-C_{1} \quad \text { for all } A \in M^{m \times n},
$$

for constants $C_{0}>0, C_{1}$ and $p>n$.

(2) Are there useful conditions guaranteeing that any minimizer $\bar{y}$ of (6.1) belongs to $C^{\infty}\left(\Omega ; \mathbf{R}^{m}\right)$ (or $\left.C^{\infty}\left(\bar{\Omega} ; \mathbf{R}^{m}\right)\right)$ ? Possible supplementary conditions (both satisfied for elasticity theory) are low values of $m$ and $n$ and invariance of the integrand under some group.

Ideally one would like not only conditions implying regularity, but a classification of possible singularities that can otherwise occur, with associated hypotheses that eliminate them.

7. The future. Having charted a selective path from the beginning of the modern era of the calculus of variations to the present day, it is natural, but no doubt rash, to speculate about what the future may hold for the subject. In the medium term one could hope for a substantially increased understanding of the multi-dimensional theory and especially of quasiconvexity, and a penetration of the newly-developed methods into other branches of mathematics. For example, one might conjecture that quasiconvexity could have a role to play in topology, since the class of quasiconvex functions contains more information about gradients than does the class of null Lagrangians, and these are used to define various topological invariants. One could also hope to better appreciate the relation between the dynamics of infinite-dimensional systems with a Lyapunov function, and the corresponding variational problem of minimizing the Lyapunov function over the state space. Most physical applications of the calculus of variations have such an origin, and there are fundamental issues that have not been sufficiently explored concerning the relationship between different kinds of local minimizers and their dynamical stability properties, and the extent to which dynamical solutions generate minimizing sequences.

Thinking further ahead, there is a general lesson to be drawn from the history of the subject. There have been periods in which the calculus of variations has developed successfully more or less as a branch of pure mathematics. Examples are the development of field theory by Weierstrass and Hilbert, and of the direct method by Tonelli and his school. However, these periods came to an end as the techniques being studied were worked out. An example is the demise of the Chicago school, much of whose fine work 
finally lacked direction. Listen to the words of one of its members, E. J. McShane [85], written in 1978:

"The problem of Bolza was the most general of the single-integral problems of the calculus of variations. Its mastery gave us the power to answer many deep and complicated questions that no one was asking. The whole subject was introverted. We who were working in it were striving to advance the theory of the calculus of variations as an end in itself, without attention to its relation with other fields of activity."

The necessary injection of ideas from outside was provided to McShane and others by the newly emerging problems of control theory. Morrey's basic work on the multidimensional theory languished unappreciated for many years, until its importance for elasticity theory became apparent. Currently we are fortunate to be in a period in which the problems of materials science are posing fundamental new questions. But this period too will come to a natural end, and we will have to wait for new questions posed by nature to push the subject forward again, just as the problem of the brachistochrone did 300 years ago.

Acknowledgments. This paper is based on a lecture given at the Conference to celebrate the 50th anniversary of the Division of Applied Mathematics at Brown University. The time I spent in the Division as a postdoc had a major influence on my mathematical career, and I am very grateful to the organisers of the conference for the opportunity to participate in the celebrations. An earlier version of the lecture was given at the Isaac Newton Institute, Cambridge, on the occasion of the 300th anniversary of Newton's solution of the brachistochrone problem, and is available as a video [4]. I am grateful to P. Loewen and J. Troutman for useful references, and to Jan Kristensen for suggesting various improvements.

\section{REFERENCES}

[1] R. Abeyaratne, C. Chu, and R. D. James, Kinetics of materials with wiggly energies: Theory and application to the evolution of twinning microstructures in a Cu-Al-Ni shape-memory alloy, Phil. Mag. A 73, 457-497 (1996)

[2] E. Acerbi and N. Fusco, Semicontinuity problems in the calculus of variations, Arch. Rat. Mech. Anal. 86, 125-145 (1984)

[3] E. J. Balder, Lectures on Young measures, Cahiers de Mathématiques de la Décision of CEREMADE, 1995

[4] J. M. Ball, The calculus of variations after 300 years, Video, obtainable from Isaac Newton Institute, Cambridge

[5] J. M. Ball, Convexity conditions and existence theorems in nonlinear elasticity, Arch. Rat. Mech. Anal. 63, 337-403 (1977)

[6] J. M. Ball, Global invertibility of Sobolev functions and the interpenetration of matter, Proc. Royal Soc. Edinburgh, 88A, 315-328 (1981)

[7] J. M. Ball, Does rank-one convexity imply quasiconvexity?, In Metastability and Incompletely Posed Problems, volume 3, pp. 17-32. IMA volumes in Mathematics and its Applications, 1987

[8] J. M. Ball, A version of the fundamental theorem for Young measures, In M. Rascle, D. Serre, and M. Slemrod, editors, Proceedings of conference on "Partial differential equations and continuum models of phase transitions", pp. 3-16. Springer Lecture Notes in Physics, No. 359, 1989

[9] J. M. Ball, Dynamics and minimizing sequences, In K. Kirchgässner, editor, Problems involving change of type, pp. 3-16. Springer Lecture Notes in Physics, no. 359, Springer-Verlag, New York, Heidelberg, Berlin, 1990 
[10] J. M. Ball, Dynamic energy minimization and phase transformations in solids, In Proceedings of ICIAM 91, SIAM, 1992

[11] J. M. Ball and C. Carstensen, Hadamard's compatibility condition for microstructures, to appear

[12] J. M. Ball and C. Carstensen, Nonclassical austenite-martensite interfaces, J. de Physique IV, to appear

[13] J. M. Ball, C. Chu, and R. D. James, Metastability and martensite, in preparation

[14] J. M. Ball, C. Chu, and R. D. James, Hysteresis during stress-induced variant rearrangement, J. de Physique IV, C8, 245-251 (1995)

[15] J. M. Ball, J. C. Currie, and P. J. Olver, Null Lagrangians, weak continuity, and variational problems of arbitrary order, J. Functional Anal. 41, 135-174 (1981)

[16] J. M. Ball, P. J. Holmes, R. D. James, R. L. Pego, and P. J. Swart, On the dynamics of fine structure, J. Nonlinear Sci. 1, 17-90 (1991)

[17] J. M. Ball and R. D. James, From Microscales to Macroscales in Materials, book in preparation

[18] J. M. Ball and R. D. James, The fundamental sufficiency theorem of the one-dimensional calculus of variations, in preparation

[19] J. M. Ball and R. D. James, Young measure disjoint sets of gradients and metastability, in preparation

[20] J. M. Ball and R. D. James, Fine phase mixtures as minimizers of energy, Arch. Rat. Mech. Anal. 100, 13-52 (1987)

[21] J. M. Ball and R. D. James, A characterization of plane strain, Proc. Roy. Soc. London A 432, 93-99 (1991)

[22] J. M. Ball and R. D. James, Proposed experimental tests of a theory of fine microstructure, and the two-well problem, Phil. Trans. Roy. Soc. London A 338, 389-450 (1992)

[23] J. M. Ball and G. Knowles, A numerical method for detecting singular minimizers, Numerische Math. 92, 193-204 (1986)

[24] J. M. Ball and J. E. Marsden, Quasiconvexity at the boundary, positivity of the second variation, and elastic stability, Arch. Rat. Mech. Anal. 86, 251-277 (1984)

[25] J. M. Ball and V. J. Mizel, Singular minimizers for regular one-dimensional problems in the calculus of variations, Bull. Amer. Math. Soc. 11, 143-146 (1984)

[26] J. M. Ball and V. J. Mizel, One-dimensional variational problems whose minimizers do not satisfy the Euler-Lagrange equations, Arch. Rat. Mech. Anal. 90, 325-388 (1985)

[27] J. M. Ball and F. Murat, $W^{1, p}$-quasiconvexity and variational problems for multiple integrals, J. Functional Analysis 58, 225-253 (1984)

[28] J. M. Ball and N. Nadirashvili, Universal singular sets for one-dimensional variational problems, Calculus of Variations and Partial Differential Equations 1, 429-438 (1993)

[29] J. Bernoulli, Letter to Basnage de Beauval, 1697

[30] K. Bhattacharya, Wedge-like microstructure in martensites, Acta Metallurgica et Materialia 39, 2431-2444 (1991)

[31] K. Bhattacharya Self-accommodation in martensite, Arch. Rat. Mech. Anal. 120, 201-244 (1992)

[32] K. Bhattacharya, Comparison of the geometrically nonlinear and linear theories of martensitic transformation, Continuum Mech. Thermodynamics 5, 205-242 (1993)

[33] K. Bhattacharya, N. B. Firoozye, R. D. James, and R. V. Kohn, Restrictions on microstructure, Proc. Royal Soc. Edinburgh 124A, 843-878 (1994)

[34] K. Bhattacharya and R. V. Kohn, Symmetry, texture and the recoverable strain of shape-memory polycrystals, Acta. Mater. 44, 529-542 (1996)

[35] K. Bhattacharya and R. V. Kohn, Elastic energy minimization and the recoverable strains of polycrystalline shape-memory materials, Arch. Rat. Mech. Anal. 139, 99-180 (1997)

[36] C. Carstensen and P. Plecháč, Adaptive algorithms for scalar non-convex variational problems, Appl. Numer. Math. 26, 203-216 (1997)

[37] C. Carstensen and P. Plecháč, Numerical solution of the scalar double-well problem allowing microstructure, Math. Comp. 6, 997-1026 (1997)

[38] L. Cesari, Optimization - Theory and Applications, Springer-Verlag, New York, 1983

[39] M. Chipot and D. Kinderlehrer, Equilibrium configurations of crystals, Arch. Rat. Mech. Anal. 103, 237-277 (1988)

[40] C. Chu, Hysteresis and microstructure: a study of biaxial loading on compound twins of copperaluminium-nickel single crystals, Ph.D. thesis, Department of Aerospace Engineering and Mechanics, University of Minnesota, 1993 
[41] F. H. Clarke and V. Zeidan, Sufficiency and the Jacobi condition in the calculus of variations, Canad. J. Math. 38, 1199-1209 (1986)

[42] C. Collins, D. Kinderlehrer, and M. Luskin, Numerical approximation of the solution of a variational problem with a double well potential, SIAM J. Numerical Analysis 28, 321-332 (1991)

[43] C. Collins and M. Luskin, Optimal-order error-estimates for the finite-element approximation of the solution of a nonconvex variational problem, Mathematics of Computation 57, 621-637 (1991)

[44] B. Dacorogna, Quasiconvexity and relaxation of nonconvex variational problems, J. Funct. Anal. 46, 102-118 (1982)

[45] B. Dacorogna, Direct Methods in the Calculus of Variations, Applied Mathematical Sciences, Vol. 78, Springer-Verlag, New York, 1989

[46] B. Dacorogna and J.-P. Haeberly, Remarks on a numerical study of convexity, quasiconvexity, and rank one convexity, In R. Serapioni and F. Tomarelli, editors, Progress in Nonlinear Differential Equations and Their Applications, volume 25, pp. 143-154, Birkhäuser, Basel, 1996, Preprint

[47] B. Dacorogna and J.-P. Haeberly, Some numerical methods for the study of the convexity notions arising in the calculus of variations, Mathematical Modelling and Numerical Analysis 32, 153-175 (1998)

[48] A. M. Davie, Singular minimizers in the calculus of variations in one dimension, Arch. Rat. Mech. Anal. 101, 161-177 (1988)

[49] E. De Giorgi, Un esempio di estremali discontinue per un problema variazionale di tipo ellittico, Boll. Un. Mat. Ital. (4) 1, 135-137 (1968)

[50] A. DeSimone, Energy minimizers for large ferromagnetic bodies, Arch. Rat. Mech. Anal. 125, 99-143 (1993)

[51] G. Dolzmann, Numerical computations of rank-one convex envelopes, to appear

[52] G. Dolzmann and S. Müller, Microstructures with finite surface energy: The two-well problem, Arch. Rat. Mech. Anal. 132, 101-141 (1995)

[53] D. T. Whiteside (ed.), The Mathematical Papers of Isaac Newton, 1697-1722, volume VIII, Cambridge University Press, 1967-1981

[54] J. L. Ericksen, Special topics in elastostatics, In C.-S. Yih, editor, Advances in Applied Mechanics, volume 17, Academic Press, 1977, pp. 189-244

[55] J. L. Ericksen, Some phase transitions in crystals, Arch. Rat. Mech. Anal. 73, 99-124 (1980)

[56] J. L. Ericksen, Some surface defects in unstressed thermoelastic solids, Arch. Rat. Mech. Anal. 88, 337-345 (1985)

[57] J. L. Ericksen, Constitutive theory for some constrained elastic crystals, Internat. J. Solids Structures 22, 951-964 (1986)

[58] J. L. Ericksen, Equilibrium theory for X-ray observations of crystals, Arch. Rat. Mech. Anal. 139, 181-200 (1997)

[59] L. C. Evans, Quasiconvexity and partial regularity in the calculus of variations, Arch. Rat. Mech. Anal. 95, 227-268 (1986)

[60] I. Fonseca, The lower quasiconvex envelope of the stored energy function of an elastic crystal, J. Math. Pures Appl. 67, 175-195 (1988)

[61] I. Fonseca, Interfacial energy and the Maxwell rule, Arch. Rat. Mech. Anal. 106, 63-95 (1989)

[62] G. Friesecke and J. B. McLeod, Dynamics as a mechanism preventing the formation of finer and finer microstructure, Arch. Rat. Mech. Anal. 133, 199-247 (1996)

[63] E. Giusti and M. Miranda, Un esempio di soluzioni discontinue per un problema di minimo relativo ad un integrale regolare del calcolo delle variazioni, Boll. Un. Mat. Ital. 2, 1-8 (1968)

[64] M. E. Gurtin, Two-phase deformations of elastic solids, Arch. Rat. Mech. Anal. 84, 1-29 (1983)

[65] K. Hane, Microstructures in thermoelastic martensites, Ph.D. thesis, Department of Aerospace Engineering and Mechanics, University of Minnesota, 1997

[66] M. R. Hestenes, Sufficient conditions for multiple integral problems in the calculus of variations, Amer. J. Math. 70, 239-275 (1948)

[67] M. R. Hestenes, Calculus of Variations and Optimal Control Theory, Wiley, 1966

[68] R. D. James, Finite deformation by mechanical twinning, Arch. Rat. Mech. Anal. 77, 143-176 (1981)

[69] R. D. James and D. Kinderlehrer, Frustration in ferromagnetic materials, Continuum Mech. Thermodynamics 2, 215-239 (1990)

[70] R. D. James and D. Kinderlehrer, Theory of magnetostriction with applications to $T b_{x} D y_{1-x} \mathrm{Fe}_{2}$, Phil. Mag. B 68, 237-274 (1993) 
[71] D. Kinderlehrer, Remarks about equilibrium configurations of crystals, In J. M. Ball, editor, Material instabilities in continuum mechanics and related mathematical problems, Oxford University Press, 1988, pp. 217-242

[72] D. Kinderlehrer and P. Pedregal, Characterizations of Young measures generated by gradients, Arch. Rat. Mech. Anal. 115, 329-365 (1991)

[73] D. Kinderlehrer and P. Pedregal, Weak-convergence of integrands and the Young measure representation, SIAM J. Math. Anal. 23, 1-19 (1991)

[74] R. V. Kohn, The relaxation of a double-well energy, Continuum Mechanics and Thermodynamics 3, 193-236 (1991)

[75] R. V. Kohn and S. Müller, Branching of twins near an austenite/twinned-martensite interface transformation, Phil. Mag. Ser. A 66, 697-715 (1992)

[76] R. V. Kohn and S. Müller, Surface energy and microstructure in coherent phase transitions, Comm. Pure Appl. Math. 47, 405-435 (1994)

[77] P. Kosmol, Bemerkungen zur brachistochrone, Abh. Math. Univ. Sem. Hamburg, 1984, pp. 91-94

[78] J. Kristensen, On the non-locality of quasiconvexity, Ann. Inst. Henri Poincaré-Analyse Nonlinéaire, 1998, to appear

[79] M. Lavrentiev, Sur quelques problèmes du calcul des variations, Ann. Mat. Pura Appl. 4, 7-28 (1926)

[80] Z. Li, Element removal method for singular minimizers in variational problems involving Lavrentiev phenomenon, Proc. Roy. Soc. Lond. A 439, 131-137 (1992)

[81] Z. Li, A numerical method for computing singular minimizers, Numer. Math. 71, 317-330 (1995)

[82] M. Luskin, On the computation of crystalline microstructure, Acta Numerica 5, 191-258 (1996)

[83] B. Manià, Sopra un esempio di Lavrentieff, Bull. Un. Mat. Ital. 13, 36-41 (1934)

[84] J. P. Matos, Young measures and the absence of fine microstructure in a class of phase transitions, European J. Applied Math. 3, 31-54 (1992)

[85] E. J. McShane, The calculus of variations from the beginning through optimal control theory, In A. B. Schwarzkopf, W. G. Kelley, and S. B. Eliason, editors, Optimal control and differential equations, Academic Press, New York, 1978, pp. 3-49

[86] N. G. Meyers, Quasi-convexity and lower semicontinuity of multiple variational integrals of any order, Trans. Amer. Math. Soc. 119, 225-249 (1965)

[87] C. B. Morrey, Quasi-convexity and the lower semicontinuity of multiple integrals, Pacific J. Math. 2, 25-53 (1952)

[88] C. B. Morrey, Multiple Integrals in the Calculus of Variations, Springer-Verlag, 1966

[89] S. Müller, Variational methods for microstructure and phase transitions, Lectures of C.I.M.E summer school, to appear

[90] S. Müller and V. Šverák, Attainment results for the two-well problem by convex integration, In J. Jost, editor, Geometric analysis and the calculus of variations, International Press, 1996, pp. 239-251.

[91] J. Nečas, Example of an irregular solution to a nonlinear elliptic system with analytic coeffcients and conditions for regularity, In Theory of Non Linear Operators, Abhandlungen Akad. der Wissen. der DDR, 1977, pp. 197-206, Proceedings of a Summer School held in Berlin, 1975

[92] G. P. Parry, On the planar rank-one convexity condition, Proc. Royal Soc. Edinburgh 125A, 247-264 (1995)

[93] P. Pedregal, Some remarks on quasiconvexity and rank-one convexity, Proc. Royal Soc. Edinburgh 126A, 1055-1065 (1996)

[94] P. Pedregal and V. Šverák, $A$ note on quasiconvexity and rank-one convexity for $2 \times 2$ matrices, J. Convex Analysis, to appear

[95] R. L. Pego, Phase transitions in one-dimensional nonlinear viscoelasticity, admissibility and stability, Arch. Rat. Mech. Anal. 97, 353-394 (1987)

[96] A. C. Pipkin, The relaxed energy density for isotropic elastic membranes, IMA Journal of Applied Mathematics 36, 85-99 (1986)

[97] A. C. Pipkin, Elastic materials with two preferred states, Quarterly J. Mech. Appl. Math. 44, 1-15 (1991)

[98] M. Pitteri, On ( $\nu+1)$-lattices, J. Elasticity 15, 3-25 (1985)

[99] M. Pitteri and G. Zanzotto, Continuum Models for Phase Transitions and Twinning in Crystals, Chapman and Hall, to appear 
[100] G. Ruddock, A microstructure of martensite which is not a minimiser of energy-the X-interface, Arch. Rat. Mech. Anal. 127, 1-39 (1994)

[101] H. J. Sussmann and J. C. Willems, 300 years of optimal control: from the brachistochrone to the maximum principle, IEEE Control Systems, June, 1997, pp. 32-44

[102] V. Šverák, Regularity properties of deformations with finite energy, Arch. Rat. Mech. Anal. 100, 105-127 (1988)

[103] V. Šverák, Rank-one convexity does not imply quasiconvexity, Proc. Royal Soc. Edinburgh 120A, 185-189 (1992)

[104] V. Šverák, On the problem of two wells, In D. Kinderlehrer, R. D. James, M. Luskin, and J. L. Ericksen, editors, Microstructure and phase transition, volume 54 of IMA Vol. Appl. Math, Springer-Verlag, New York, 1993, pp. 183-189

[105] M. A. Sychev, On the regularity of solutions of some variational problems, Soviet Math. Dokl. 43, 292-296 (1991)

[106] M. A. Sychev, On a classical problem of the calculus of variations, Soviet Math. Dokl. 44, 116-120 (1992)

[107] M. A. Sychev, Lebesgue measure of the universal singular set for the simplest problems in the calculus of variations, Siberian Mathematical J. 35, 1220-1233 (1994)

[108] A. Taheri, Local minimizers in the calculus of variations, Ph.D. thesis, Department of Mathematics, Heriot-Watt University, 1997

[109] L. Tartar, The compensated compactness method applied to systems of conservation laws, In J. M. Ball, editor, Systems of Nonlinear Partial Differential Equations, pp. 263-285. NATO Adv. Sci. Inst. Series, Vol. C111, Reidel, Dordrecht-Boston, 1982

[110] L. Tonelli, Fondamenti di Calcolo delle Variazioni, Volumes I, II. Zanichelli, 1921-1923

[111] L. Tonelli, Sugli integrali del calcolo delle variazioni in forma ordinaria, Ann. R. Scuola Norm. Sup. Pisa 21, 289-293 (1934), in L. Tonelli, Opere Scelte, Vol. III, \# 105, Edizioni Cremonese, Roma, 1961

[112] J. L. Troutman, Variational Calculus and Optimal Control, Springer-Verlag, New York, 1996

[113] L. C. Young, Lectures on the Calculus of Variations and Optimal Control Theory, Saunders, 1969. Reprinted by Chelsea, 1980

[114] G. Zanzotto, On the material symmetry group of elastic crystals and the Born rule, Arch. Rat. Mech. Anal. 121, 1-36 (1992)

[115] K. Zhang, On stability of quasiconvex hulls, in Calculus of Variations and Partial Differential Equations, to appear

[116] K. Zhang, On the structure of quasiconvex hulls, Ann. Inst. Henri Poincaré - Analyse non linéaire, to appear

[117] K. Zhang, A construction of quasiconvex functions with linear growth at infinity, Ann. Scuola Norm. Sup. Pisa Cl. Sci. (4) 19, 313-326 (1992) 